\title{
Frequency Map Analysis of Nonlinear Dynamics in the NLC Main Damping Rings
}

\author{
A. Wolski, M. Venturini, S. Marks, W. Wan \\ Lawrence Berkeley National Laboratory, Berkeley, California, 94720.
}

October $11^{\text {th }}, 2004$

\begin{abstract}
To avoid radiation damage, the acceptance of linear collider damping rings must be large enough that injection efficiency close to $100 \%$ can be achieved. Survival plots based on tracking particles in the NLC Main Damping Ring lattice suggest a dynamic aperture with some margin over the specified injected beam size and energy spread [1]. Here, we apply Frequency Map Analysis to give a more detailed picture of the dynamical stability of particle trajectories in the presence of lattice nonlinearities arising from the sextupoles and the damping wiggler. The techniques that we use are of general applicability to nonlinear elements in beamlines, and in particular will be used for analysis of wiggler effects in future damping ring designs.
\end{abstract}

\section{Disclaimer}

This document was prepared as an account of work sponsored by the United States Government. While this document is believed to contain correct information, neither the United States Government nor any agency thereof, nor The Regents of the University of California, nor any of their employees, makes any warranty, express or implied, or assumes any legal responsibility for the accuracy, completeness, or usefulness of any information, apparatus, product, or process disclosed, or represents that its use would not infringe privately owned rights. Reference herein to any specific commercial product, process, or service by its trade name, trademark, manufacturer, or otherwise, does not necessarily constitute or imply its endorsement, recommendation, or favoring by the United States Government or any agency thereof, or The Regents of the University of California. The views and opinions of authors expressed herein do not necessarily state or reflect those of the United States Government or any agency thereof or The Regents of the University of California.

LBNL is an equal opportunities employer.

This work was supported by the Director, Office of Science, High Energy Physics, U.S. Department of Energy under Contract No. DE-AC03-76SF00098. 


\section{Introduction}

Previous studies for the present NLC Main Damping Ring (MDR) indicate a dynamic aperture close to fifteen times the injected beam size in the presence of nonlinearities from the sextupoles and the damping wiggler. The results are based simply on the survival of particles over tracking a few hundred times through the lattice, with a range of betatron amplitudes and energy errors. Such studies give very limited information on the underlying dynamics. Frequency Map Analysis (FMA) provides more detailed information, for example on the resonance structure of the phase space lying within the dynamic aperture, that allows a more realistic assessment of the stability of particle trajectories, and provides some indication of approaches that may improve the dynamic aperture. FMA has been used successfully to optimize the dynamic momentum acceptance of the ALS [2], resulting in a significant improvement in the beam lifetime.

The principles of FMA are now becoming well known in the accelerator community. Briefly, a tracking code is used to determine the phase-space co-ordinates of a particle at a given "observation point" in the lattice on successive turns. A modified Fourier analysis is then applied to determine the betatron tune to accuracy $\sim 1 / N^{4}$ given $N$ turns of data. For the present studies, we have used a technique based on applying a Hanning filter to the data, and interpolating around the highest peak of the Fourier spectrum to determine the betatron tune with high resolution. This procedure gives the horizontal and vertical betatron tunes as a function of horizontal and vertical amplitude. Additional information may be obtained by tracking for a further $N$ turns, and applying the analysis to this second set of data. The difference in the tunes between the first and second sets of data indicates the "diffusion rate" in frequency space. In practice, high diffusion rates are associated with resonance lines, and under some circumstances indicate dynamics with limited stability.

In this note, we present the results of Frequency Map Analysis of the NLC MDR. The only nonlinear elements in the lattice are the sextupoles and the damping wiggler. Multipole errors are not included: although they will certainly have an adverse effect on the dynamic aperture, they are not yet fully characterized. Once information is available on the magnet errors, then the analysis can easily be repeated; it is useful, however, to present results from an "ideal" lattice for later comparison. We first describe the model used for the dynamics in the wiggler. We then present the results from two different tracking codes (MARYLIE 3.0 and MERLIN III) and see that they are in good agreement.

Regarding the model of dynamics in the wiggler, we consider only the nonlinearities that appear from the field map in the design model. Random errors that will arise in the construction of a real device have not so far been included. The parameters of the wiggler are described in reference [1]. Briefly, the nominal peak field is $2.1 \mathrm{~T}$, and the period is $27 \mathrm{~cm}$. A magnetic model of a periodic section of the wiggler was used as a basis for a study of the particle dynamics in the wiggler [3], where it was found that the wiggler nonlinearities make a significant contribution to the limit on the dynamic aperture. Since then, we discovered that inaccuracies in the field map, associated with the finite size of the mesh used in the field calculation, had a significant impact on the dynamical model derived from the field map. We have therefore refined the field model, to produce a field map that satisfies the physical 
constraints (Maxwell's equations) to better accuracy. As shall be seen, the nonlinearities in the new wiggler model have a significantly smaller impact on the dynamics in the damping ring than found in the previous wiggler model. Note that the geometry and materials of the wiggler were not changed; only the calculation of the field was improved (by refining the mesh) in producing the new field map.

The procedure for analyzing the dynamics in the damping wiggler may be summarized as follows.

1. A magnetic field map for one period of the wiggler is produced using a modeling code, e.g. TOSCA. The field map consists of a table specifying the horizontal, vertical, and longitudinal field components at each point on a rectangular grid.

2. An analytic series is fitted to the numerical field map. This reduces the field map to a set of coefficients, and ensures that the field model satisfies Maxwell's equations.

3. The analytic series is used to generate a dynamical map for a particle traveling through the wiggler. The map may be generated either by a differential algebra code (e.g. COSY) in which case it is represented by a truncated power series, or by use of a Lie algebra code (e.g. MARYLIE). The advantage of the Lie algebra code is that the map is exactly symplectic.

4. The wiggler map is used in a tracking code to generate data for the frequency map analysis. By comparing different wiggler models (including a "linear" model), the impact of the wiggler on the dynamic aperture may be estimated.

In several of these steps, there is a choice of computational tools that may be applied. It is useful to compare the results from different codes, to verify that the final results are not sensitive to different choices. In this note, we report the results of some of these comparisons, and show that very similar results are obtained, irrespective of which code is used. In the following sections, we discuss in more detail each of the steps in the above procedure, presenting the results relevant to each step. Since generating a field map using a magnetic modeling code is a routine (though not necessarily straightforward) procedure, we begin by describing the analytic fit to the field map.

\section{Magnetic Field Model}

Our aim in this section is to produce from numerical field data an analytic expression for the field, which may then be used to generate a dynamical map in either Lie form or truncated power series form. For the initial fit, we have found it best to use a cylindrical representation of the field; this may then be converted to a Cartesian representation for generating the dynamical map, if necessary.

A static magnetic field solution to Maxwell's equations, periodic in the longitudinal coordinate may be written: 


$$
\begin{aligned}
& B_{\rho}=\sum_{m n} \alpha_{m n} \mathrm{I}_{m}^{\prime}\left(n k_{z} \rho\right) \sin (m \phi) \cos \left(n k_{z} z\right) \\
& B_{\phi}=\sum_{m n} \alpha_{m n} \frac{m}{n k_{z} \rho} \mathrm{I}_{m}\left(n k_{z} \rho\right) \cos (m \phi) \cos \left(n k_{z} z\right) \\
& B_{z}=-\sum_{m n} \alpha_{m n} \mathrm{I}_{m}\left(n k_{z} \rho\right) \sin (m \phi) \sin \left(n k_{z} z\right)
\end{aligned}
$$

$\mathrm{I}_{m}(x)$ is a modified Bessel function. Given numerical field data over one periodic section, the coefficients $\alpha_{m n}$ may be found simply from a two-dimensional Fourier analysis. Specifically, we take the Fourier transform of the radial field component over the surface of a cylinder inscribed through the wiggler. Because of the exponential behavior of the modified Bessel function, fitting errors decrease towards the axis of the cylinder, and increase away from the axis. It is therefore advantageous to take the surface of the fit with as large a radius as possible; however, this depends on having field data of good quality away from the axis of the wiggler.

It is possible to generate a dynamical map directly by integration of equations (1). However, it is often convenient to convert the representation into a Cartesian form, which may be more easily handled, for example, by a differential algebra code. The corresponding Cartesian form to (1) is:

$$
\begin{aligned}
B_{x} & =-\sum_{m, n} c_{m n} \frac{m k_{x}}{k_{y, m n}} \sin \left(m k_{x} x\right) \cos \left(n k_{z} z\right) \sinh \left(k_{y, m n} y\right) \\
B_{y} & =\sum_{m, n} c_{m n} \cos \left(m k_{x} x\right) \cos \left(n k_{z} z\right) \cosh \left(k_{y, m n} y\right) \\
B_{z} & =-\sum_{m, n} c_{m n} \frac{n k_{z}}{k_{y, m n}} \cos \left(m k_{x} x\right) \sin \left(n k_{z} z\right) \sinh \left(k_{y, m n} y\right)
\end{aligned}
$$

Note that this does not correspond exactly to the cylindrical representation, since the Cartesian expansion (2) assumes that the field is periodic in the horizontal co-ordinate $x$, which is generally not the case, while the cylindrical expansion (1) reflects the necessary azimuthal periodicity. In practice, we find that taking a value of $k_{x}$ corresponding to a horizontal "period" of several times the radius of the surface used for the cylindrical fit, we may achieve an excellent Cartesian fit within the original cylinder. For a given value of $k_{x}$, the coefficients $\alpha_{m n}$ and $c_{m n}$ are related by:

$$
\alpha_{m n}=\frac{2(-1)^{\frac{m-1}{2}} m !}{\left(n k_{z}\right)^{m-1}} \sum_{m^{\prime}}\left(\sum_{q=0}^{\frac{m-1}{2}} \frac{k_{y, m^{\prime} n}^{m-2}\left(m^{\prime} k_{x}\right)^{2 q}}{(2 q) !(m-2 q) !}\right) c_{m^{\prime} n}
$$

This expression allows one to calculate a set $\alpha_{m n}$ from a given set of $c_{m n}$, or (by a matrix inversion) a set $c_{m n}$ from a given set of $\alpha_{m n}$.

The results from two wiggler models, which we refer to as model C and model D, are shown in Figure 1 and Figure 2. The field map for model D was generated using the same geometry as model $\mathrm{C}$, but with a finer mesh. In both cases, the fit was based on a cylinder of radius $6 \mathrm{~mm}$, with mode numbers up to $m=9$ (azimuthal or horizontal modes) and $n=39$ (longitudinal). 


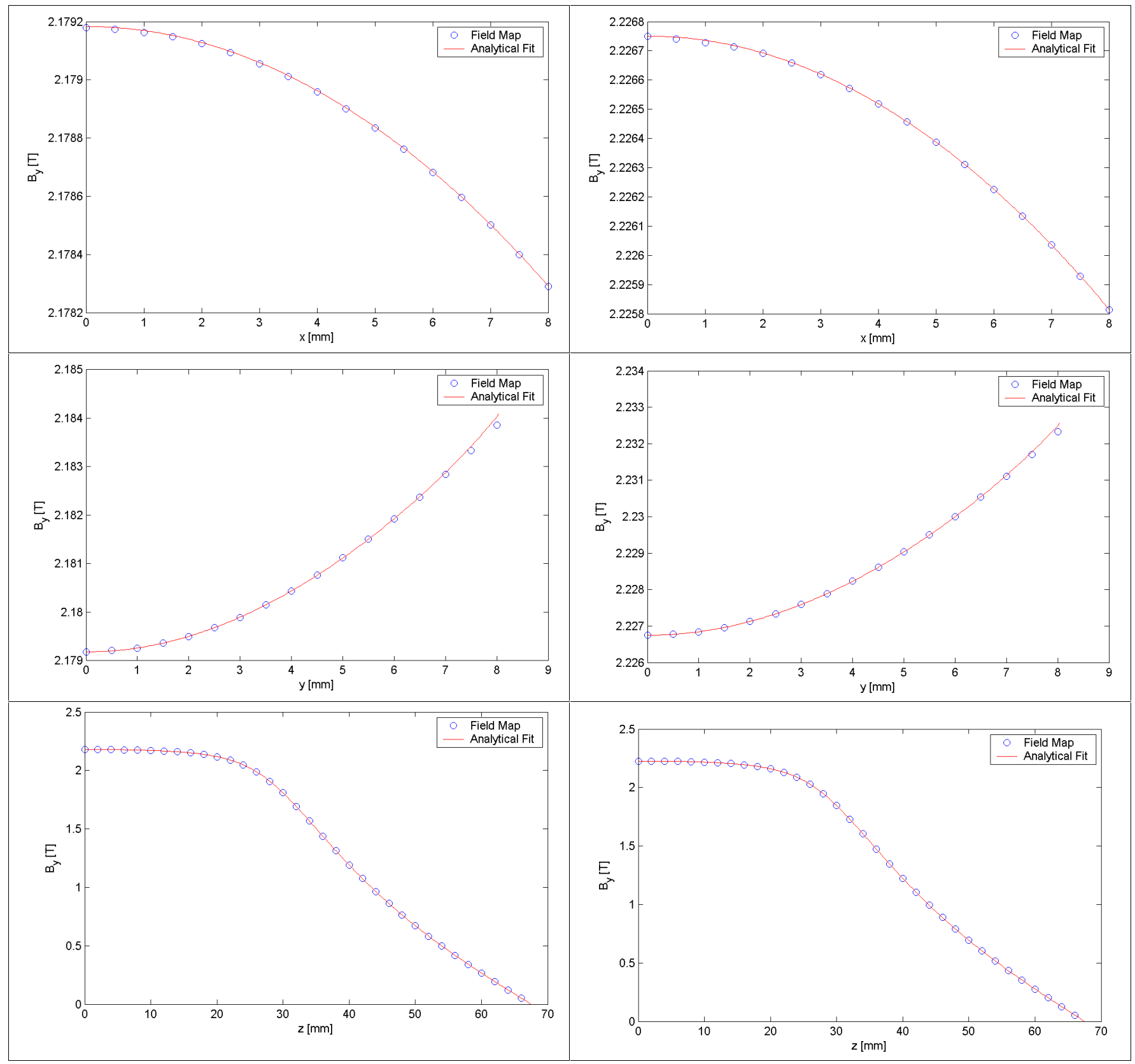

Figure 1: Horizontal, vertical and longitudinal field profiles (top, middle and bottom respectively) for two models of the NLC MDR wiggler. Model C is on the left; model D (generated with a finer mesh) is on the right. The blue circles show the numerical field data from the magnet modeling code; the red line shows the results of an analytical fit based on the Cartesian representation (2).

We note that model $\mathrm{D}$ has a peak field about $2 \%$ higher than that in model $\mathrm{C}$. On the midplane, the residuals of the fit are less than 10 gauss, or less than $0.05 \%$ of the peak field. The residuals in model $\mathrm{D}$, with the finer mesh, are nearly a factor of two smaller than those in model C. 


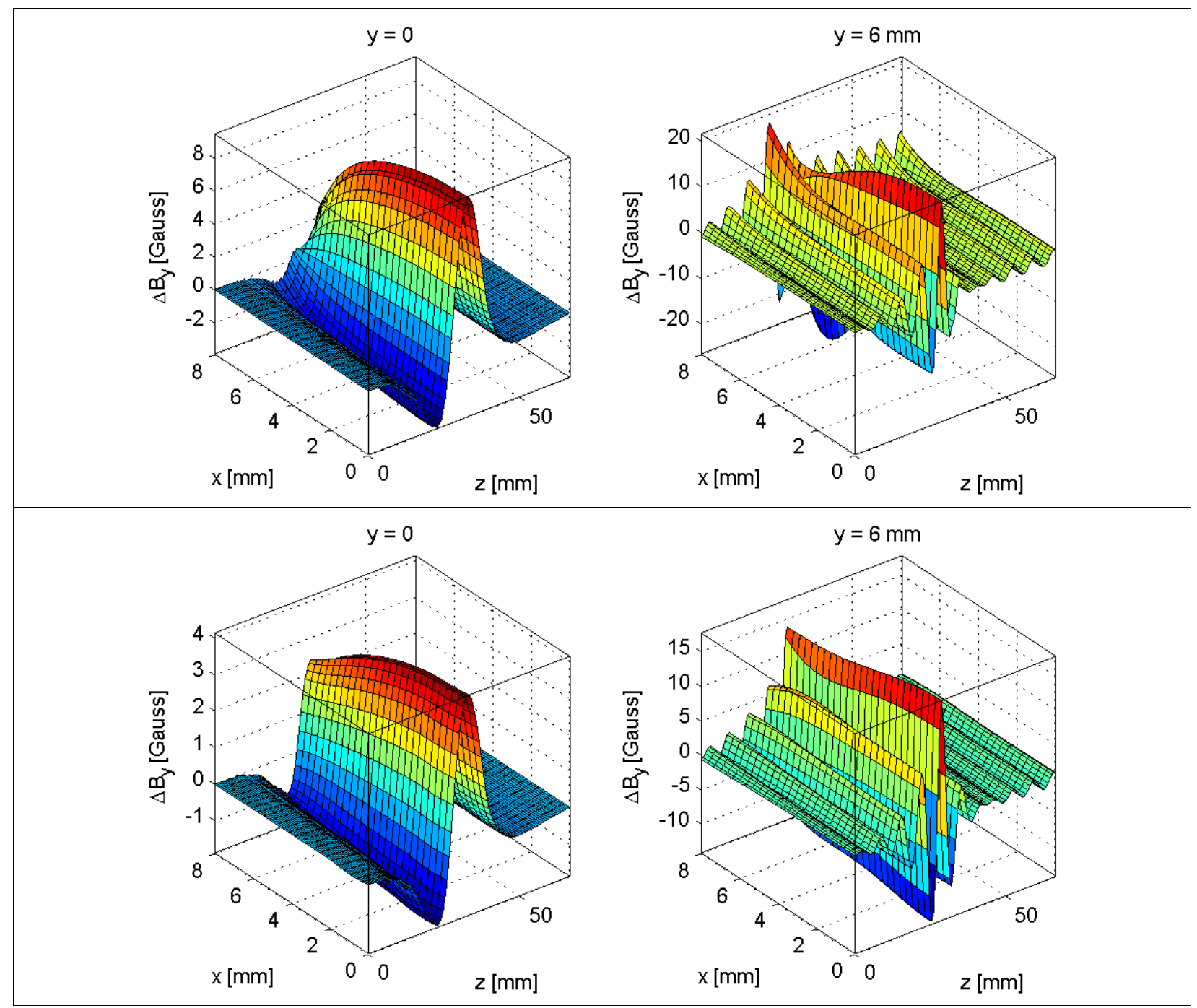

Figure 2: Residual between numerical field data and an analytical fit for wiggler model C (top) and D (bottom). The left-hand plots show the differences between the vertical field components on the midplane, and the right-hand plots show the differences on a horizontal plane $6 \mathrm{~mm}$ above the mid-plane.

\section{Generating the Dynamical Map}

Having obtained an accurate analytical expression for the field, there are a number of techniques possible for generating the dynamical map. Here, we consider the use of a differential algebra code to produce a dynamical map represented as a truncated power series, and the use of a Lie algebra code to produce a dynamical map in the form of a Lie generator.

\subsection{Truncated Power Series Map}

Use of a differential algebra codes makes it straightforward to construct a truncated power series map for the dynamics in one period of the wiggler. Essentially, one simply needs to implement an integration routine for tracking a particle through the field given either by expressions (1) or by expressions (2), and then replace the 'real' variables representing the particle's phase-space co-ordinates by 'differential algebra' variables. 
Here, we compare two different integration routines implemented in the differential algebra code COSY. The first is a Runge-Kutta integrator that integrates the equations of motion that follow from writing the Lorentz force on a particle given by the fields (1). The second is a symplectic integrator that integrates the Hamiltonian equations of motion, with the Hamiltonian obtained from the vector potential corresponding to the fields (1). The RungeKutta integrator includes all terms in the equations of motion, but there is a small 'symplectic error' from the finite step size taken in the integration. By specifying an appropriate precision for the integration, this error can be kept small. The symplectic integrator has the advantage that the map produced is symplectic (up to the order of the map), but to achieve this, the paraxial approximation is needed, which omits some terms from the Hamiltonian.

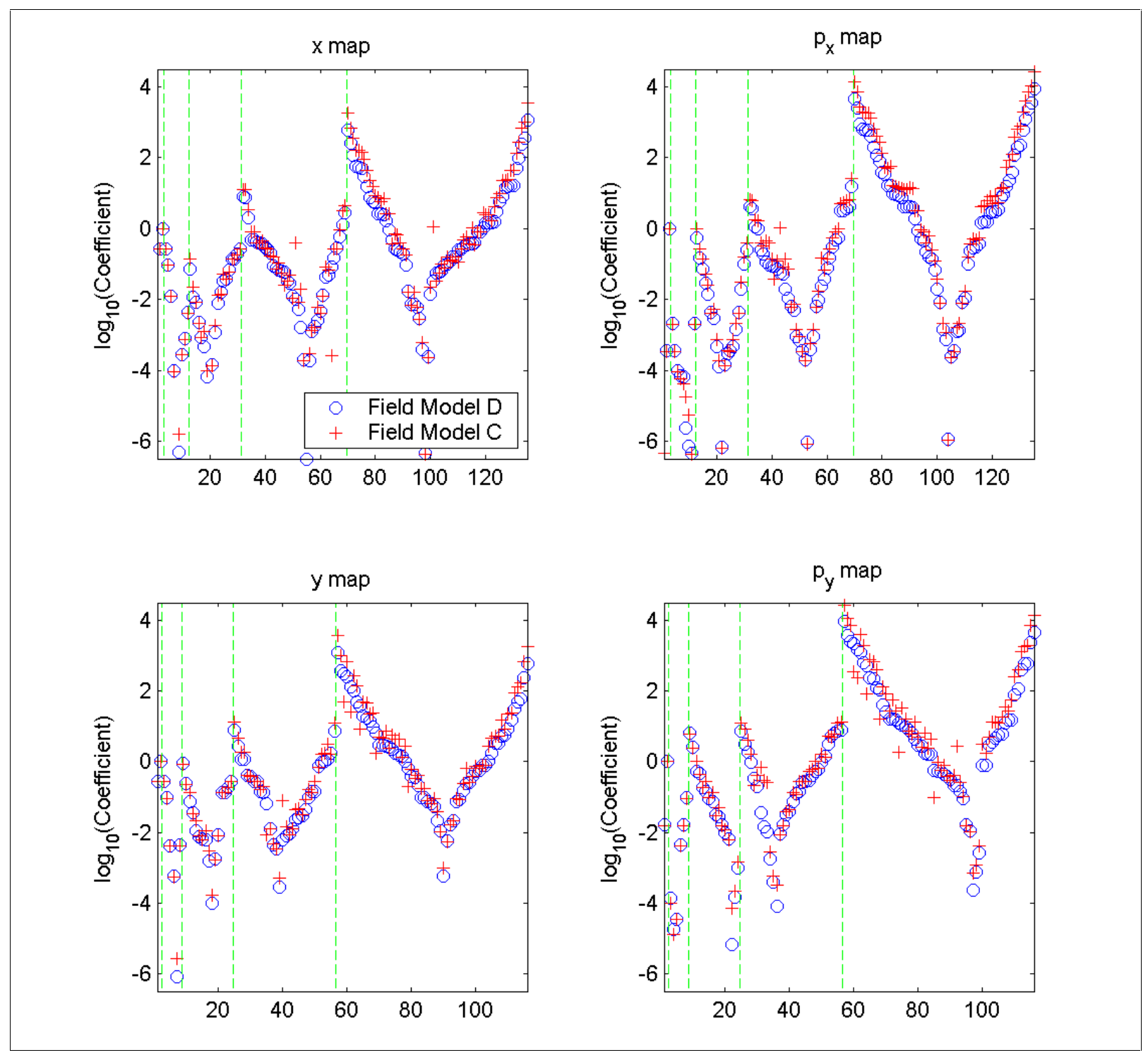

Figure 3: Comparison between coefficients in the dynamical maps for two different field models, computed using a Runge-Kutta integrator. For further explanation, see the text. 

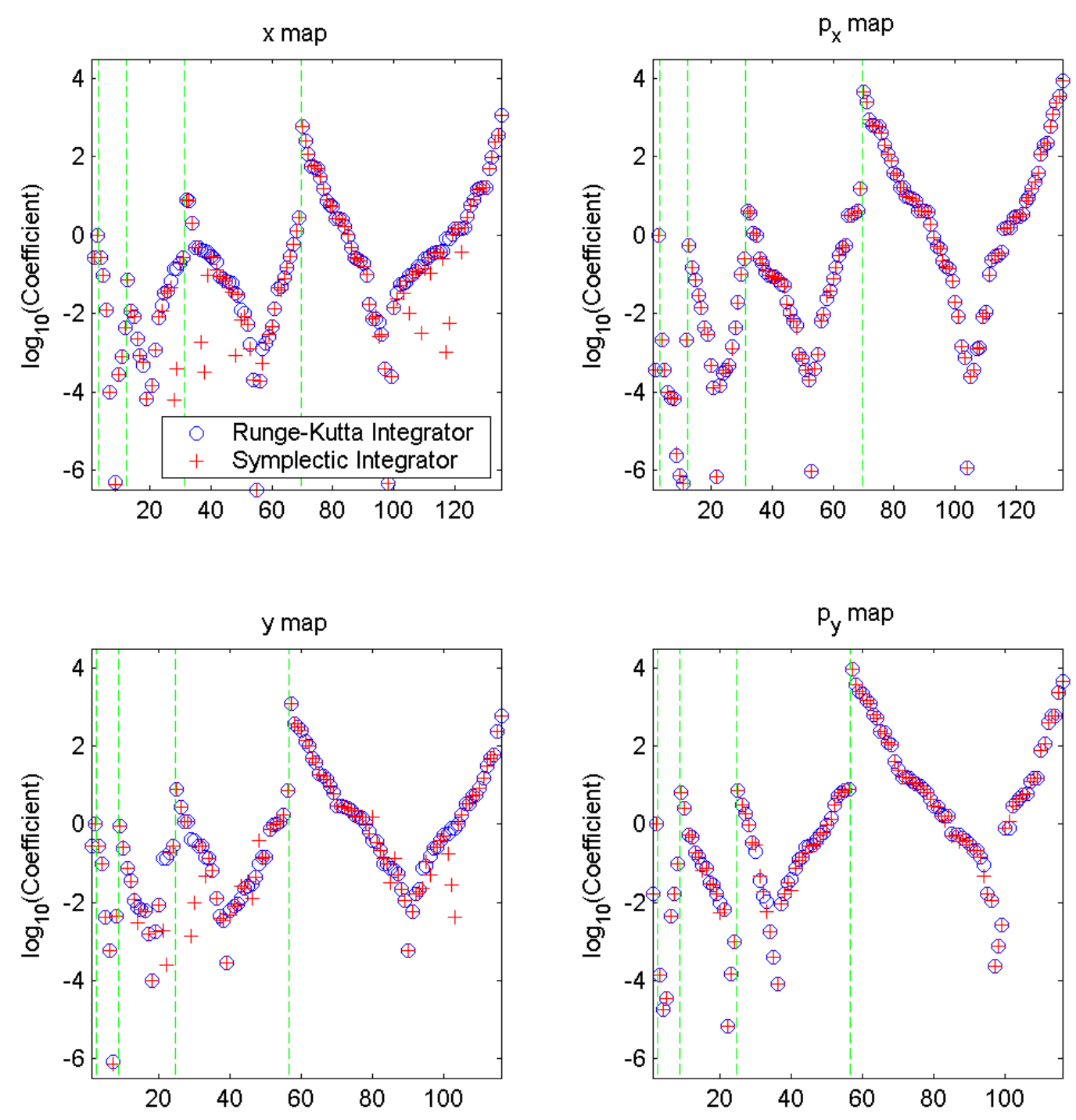

Figure 4: Comparison between coefficients in the dynamical maps for field model D, computed using two different integration methods. For further explanation, see the text.

A comparison between the maps obtained using the Runge-Kutta integrator for the two different field models is shown in Figure 3. The coefficients of the terms in the maps for the four transverse phase-space variables through one period of the wigglers are shown in increasing order on a logarithmic scale (we show the absolute values, so those coefficients on a descending slope are negative). The broken green lines separate terms of different order; in the cases shown, we calculated the maps up to fifth order. A similar comparison, but for field model D using the two different integration methods, is shown in Figure 4. Note that the blue circles in Figure 4 are the same as in Figure 3, i.e. for the map computed using a Runge-Kutta integrator for field model D. 
We see that there is good agreement between the maps produced using the two different integration methods, but somewhat larger discrepancy between the coefficients from the two different field models.

The small difference between the maps produced by the Runge-Kutta and the symplectic integrator does have some impact on the symplecticity of the map. We can verify the symplecticity by calculating:

$$
\Delta=\mathbf{J}^{\mathrm{T}} \cdot \mathbf{S} \cdot \mathbf{J}-\mathbf{S}
$$

where $\mathbf{J}$ is the Jacobian of the map, and $\mathbf{S}$ is the usual symplectic form. For a perfectly symplectic map, $\Delta$ will be exactly zero. For the truncated power series maps produced using the differential algebra code, we can obtain power series for each component of $\Delta$. The fact that the power series are truncated at some order, $n$, makes the maps non-symplectic; for a map that is symplectic to order $n$, we expect only terms of order $n$ and higher to appear in $\Delta$. Figure 5 shows (on a logarithmic scale) the components of $\Delta$ up to order 4 , evaluated for unit values of the phase-space variables, for the maps produced using the Runge-Kutta integrator and the symplectic integrator (note that $\Delta$ is antisymmetric, so has only 15 independent components). The symplectic error in the map produced using the symplectic integrator is clearly smaller than that in the map produced using the Runge-Kutta integrator. The increasing errors in the terms of higher order are consistent with numerical errors arising from the cancellation of terms with large coefficients.
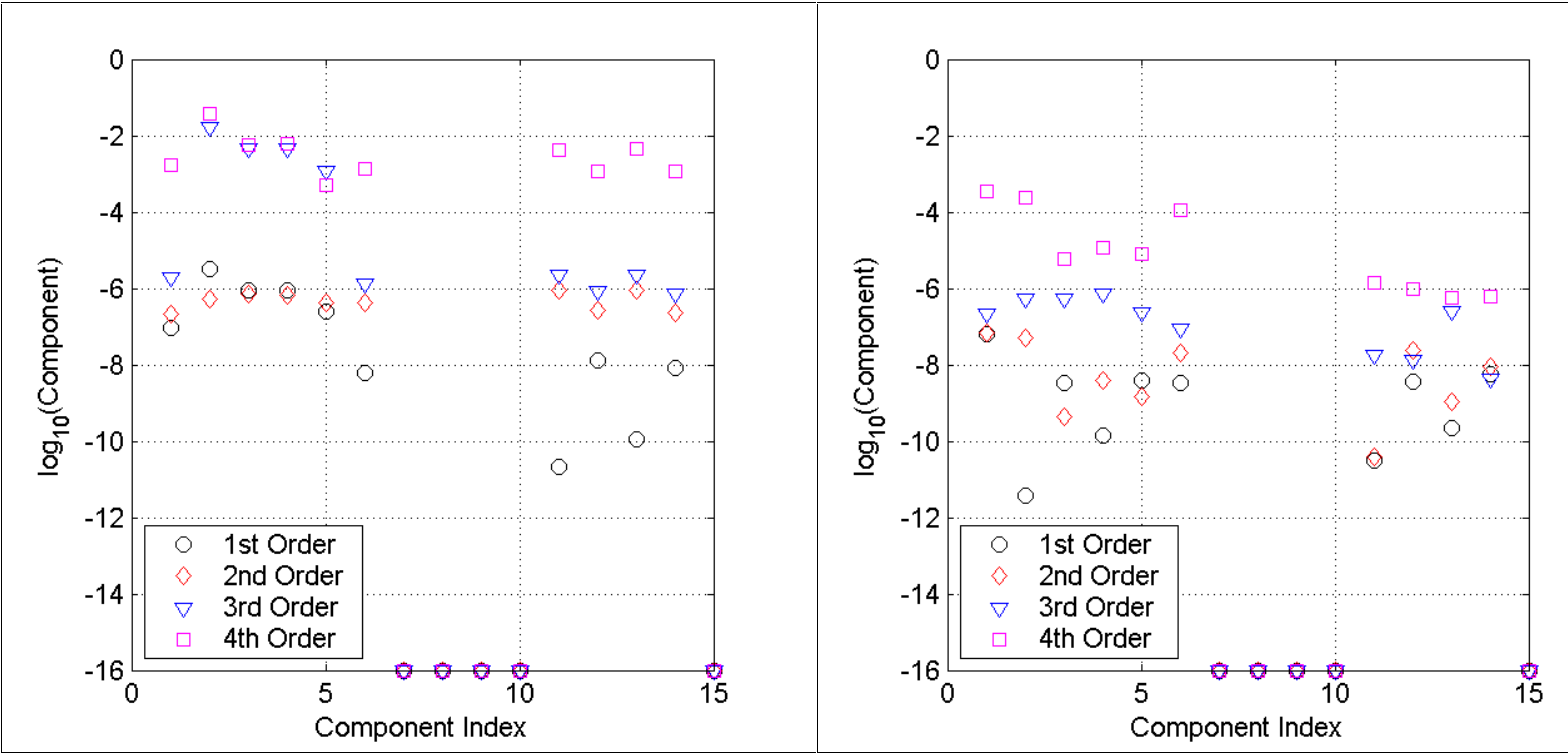

Figure 5: Symplectic error in the truncated power series maps produced using the differential algebra code with (left) the Runge-Kutta integrator, and (right) the symplectic integrator. The absolute values of each component of $\Delta$, evaluating terms of a given order with unity values for the phase-space variables, are plotted on a logarithmic scale.

For both integrators, the symplectic error appears small enough that we do not expect to see any significant non-symplectic effects in the dynamics in the damping ring. 


\subsection{Lie Algebra Map}

As an alternative to truncated power series, one can use Lie generators to represent transfer maps. Lie maps provide some advantages: a truncation of a Lie map through a given order in the Lie generators preserves symplecticity and the number of parameters needed to specify a Lie map is smaller than that required for an equivalent truncated power series. A disadvantage is the labor needed for encoding, which increases considerably with the order of the map. By contrast, in a code that manipulates truncated power series, the order of a map is just a parameter. Presently, in the code MARYLIE, calculation and manipulation of Lie maps are limited to third order (i.e. fourth order in the Lie generators). Although a higher-order version of the code is available it is still incomplete and was not used in this study.

A Lie map through a specified magnetic field is calculated by solving the canonical equations for each Lie generator. This requires an expansion of the Hamiltonian as a power series, including the vector potential corresponding to the magnetic field (1). Unlike the map resulting from the symplectic integrator discussed in the previous section, the map obtained using MARYLIE is exact (through third order). A detailed outline of the calculation is reported in [3].
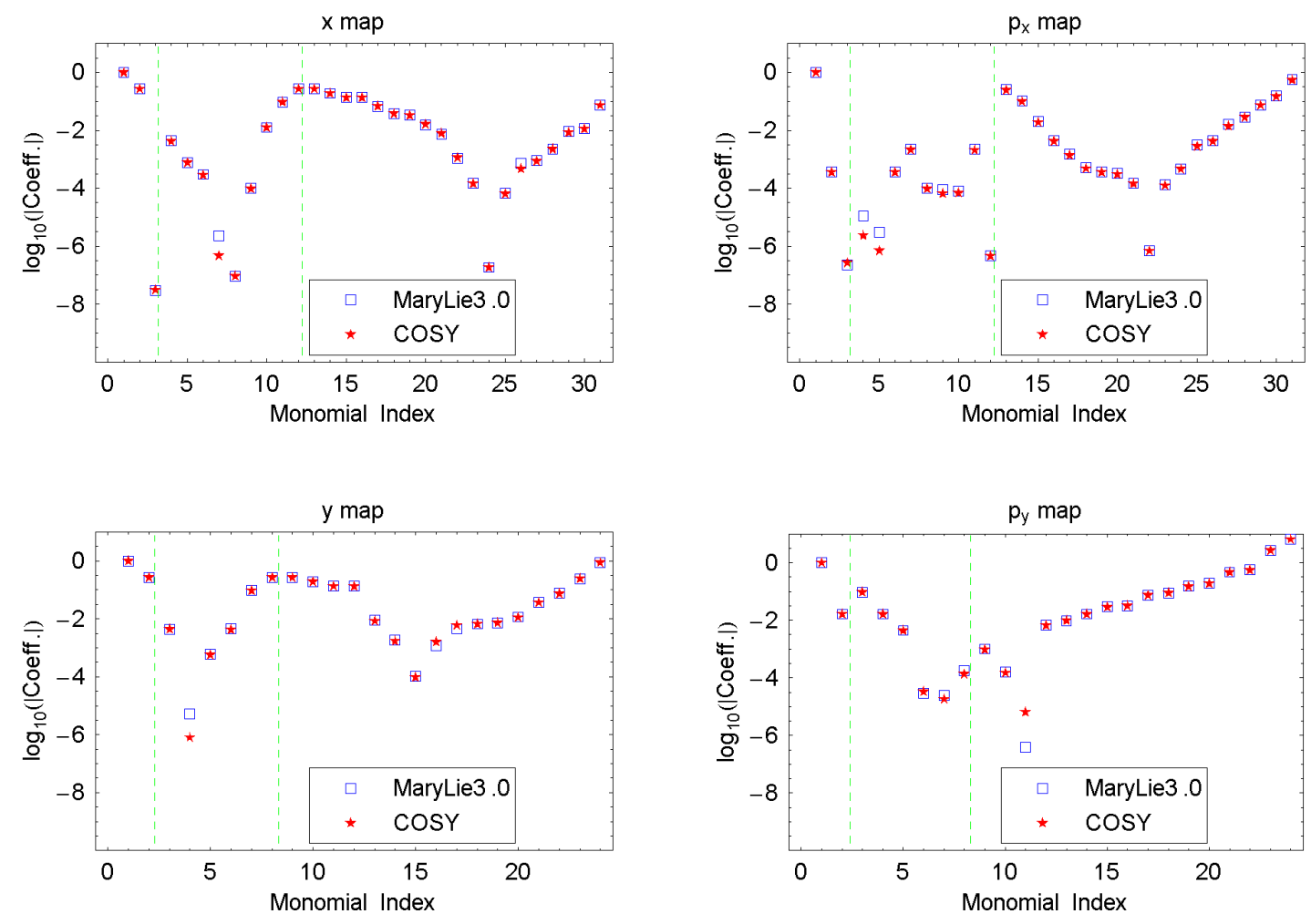

Figure 6: Comparison between coefficients in the dynamical maps for field model D as computed using MARYLIE3.0 and COSY (Runge-Kutta integrator).

A comparison between the coefficients of the transfer map for a wiggler period as calculated using the Runge-Kutta method and the Lie generator method is shown in Figure 6. For 
consistency in the case of the Lie map we report (the logarithm of) the coefficients of the corresponding truncated Taylor series map, which is obtained from the Lie generators using standard manipulation routines implemented in MARYLIE. The transfer map for the wiggler period in both cases starts at the center of the poles. For most coefficients the agreement is good; where there is some discrepancy, the coefficients are small and (we hope) not important for the dynamics.

\section{Frequency Map Analysis}

We have carried out Frequency Map Analysis for the NLC Main Damping Ring lattice in six different cases, shown in Table 1. The cases were chosen to allow comparison of results between different field models and different orders for the dynamical map. The dynamics are dominated by the sextupoles; to isolate the effects of the wiggler, the frequency maps for the two different field models are compared with the sextupoles off. Each of the six cases was analyzed with the truncated power series map computed with COSY, and in some selected cases with the Lie algebra map computed with MARYLIE. The results from the MARYLIE calculation (which is limited to third order) are reported in the Appendix.

Table 1: Cases considered in Frequency Map Analysis of the NLC Main Damping Ring lattice.

\begin{tabular}{|c|c|c|c|c|}
\hline Case Number & Field Model & Dynamical Map Order & Sextupoles & Variables \\
\hline \hline 1 & D & 5 & Off & $\mathrm{x}-\mathrm{y}$ \\
\hline 2 & C & 5 & Off & $\mathrm{x}-\mathrm{y}$ \\
\hline 3 & D & 5 & On & $\mathrm{x}-\mathrm{y}$ \\
\hline 4 & D & 3 & On & $\mathrm{x}-\mathrm{y}$ \\
\hline 5 & D & 1 & On & $\mathrm{x}-\mathrm{y}$ \\
\hline 6 & D & 5 & On & $\mathrm{x}-\delta$ \\
\hline
\end{tabular}

In tracking to produce the data for Frequency Map Analysis, we consistently use a lattice in which each full period of the wiggler is replaced by a dynamical map. The structure of one section of wiggler is shown in Figure 7. Each section consists of six full periods, plus end pieces designed such that:

- The total field integral through the wiggler is zero.

- The trajectory of a particle entering the wiggler along the magnetic axis is centered on the magnetic axis.

- A particle entering the wiggler along the magnetic axis exits the wiggler along the magnetic axis.

Only full periods of the wiggler are represented by nonlinear map, and we have not included any effects from nonlinearities in the ends of the wiggler. However, a magnetic field map has been produced only for a full period; the nonlinear fields in the ends are not known at this time. Since the ends are short compared to the body of the wiggler, we hope that the nonlinear effects from the ends will not significantly affect our results. 


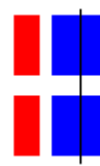

Figure 7: Structure of one section of the NLC MDR wiggler. The different color blocks represent poles bending the beam in opposite directions. The black lines divide full periods of the wiggler, represented by a dynamical map for tracking.

There are 6 full periods per wiggler section, and 32 wiggler sections in the complete lattice. It is of course possible to produce a map for 6 periods directly from the map from a single period; although some loss of high order effects may be expected, this could speed tracking. Since data for a Frequency Map Analysis may still be obtained in a few hours (using a $5^{\text {th }}$ order map for each wiggler period) with each period represented by a separate map, we have chosen not to concatenate maps for wiggler periods.

The tune shifts with amplitude can be used as a simple indicator of the effect of the wiggler on the dynamics. These are found from the Frequency Map Analysis. The horizontal and vertical tunes vs horizontal and vertical action in Cases 1, 2, 3 and 5 are shown in Figure 8, and numerical values are given in Table 2.

Table 2: Tune shifts with amplitudes for Cases 1, 2, 3 and 5, using the wiggler field map computed with COSY. The numerical quantities are the gradients of the lines shown in Figure 8.

\begin{tabular}{|c|c|c|c|c|c|c|c|}
\hline Case & Model & Map Order & Sextupoles & $\frac{\partial v_{x}}{\partial J_{x}}\left[\mathrm{~nm}^{-1}\right]$ & $\frac{\partial v_{x}}{\partial J_{y}}\left[\mathrm{~nm}^{-1}\right]$ & $\frac{\partial v_{y}}{\partial J_{x}}\left[\mathrm{~nm}^{-1}\right]$ & $\frac{\partial v_{y}}{\partial J_{y}}\left[\mathrm{~nm}^{-1}\right]$ \\
\hline \hline 1 & D & 5 & Off & -0.0775 & 0.229 & 0.214 & 8.04 \\
\hline 2 & C & 5 & Off & -0.144 & 0.465 & 0.423 & 8.74 \\
\hline 3 & D & 5 & On & -3.67 & 24.3 & 23.1 & 36.2 \\
\hline 5 & D & 1 & On & -3.59 & 24.0 & 22.9 & 28.1 \\
\hline
\end{tabular}

The tune shifts are clearly dominated by the sextupoles. The wiggler itself makes a small contribution to the tune shifts; the most significant contribution is to the vertical tune shift with vertical amplitude. There appears to be some difference between wiggler models $\mathrm{C}$ and $\mathrm{D}$, which may be of concern (the only difference between them is the mesh density in calculating the field map), except that the tune shifts are mostly so small that the values calculated may be at the limit of the accuracy that may be reasonable expected from the techniques we are using. For the largest tune shift, in the vertical plane, there is better agreement between the models (at the level of 10\%) than for the smaller tune shifts (where the discrepancy is of the order of a factor of 2).

Plots in co-ordinate space and in tune space for the different cases are shown in Figure 9 through Figure 20. Note that MARYLIE3.0 can be used both to compute the map through the wiggler, and to track through the lattice. MERLIN was used to track the lattice with the wiggler map computed with COSY. At the observation point, the beta functions are $16.0 \mathrm{~m}$ horizontally, and $1.57 \mathrm{~m}$ vertically. With an injected beam normalized emittance of $150 \mu \mathrm{m}$, 
the rms beam sizes are $787 \mu \mathrm{m}$ horizontally and $247 \mu \mathrm{m}$ vertically. Note that we plot separately the frequency maps for positive and negative values of the co-ordinates. The larger tune shifts from wiggler model $\mathrm{C}$ are apparent in some reduction of the dynamic aperture with the sextupoles turned off (compare Figure 9 with Figure 11), but the dynamic aperture in the case of wiggler model D and the sextupoles turned off is of the order 100 times the beam size horizontally, and 50 times the beam size vertically (counting all stable orbits within the dynamic aperture). At these large amplitudes, we might expect some sensitivity to small nonlinear terms.

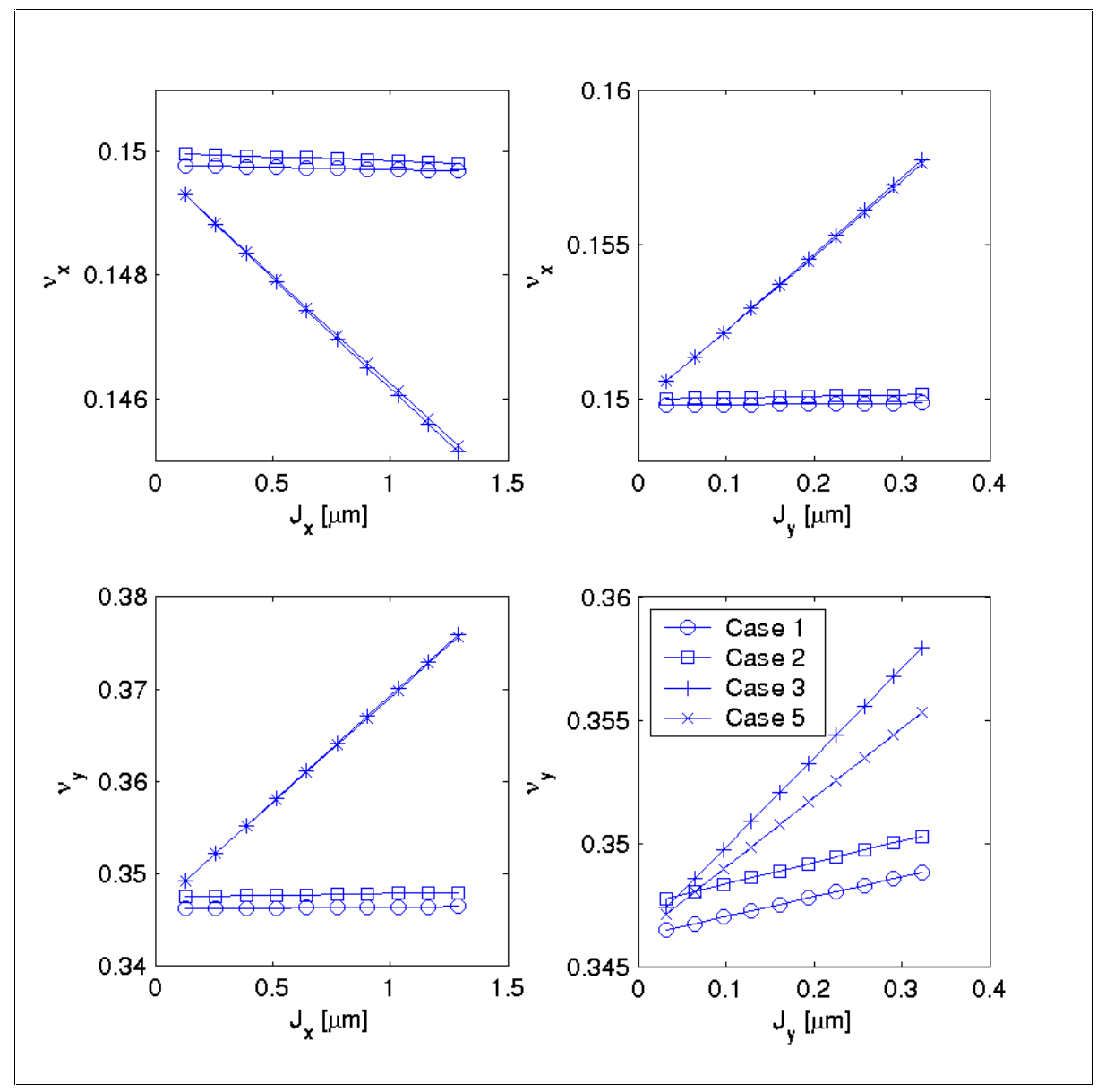

Figure 8: Tune shifts with amplitude. Cases 1 and 2: sextupoles off; cases 3 and 5: sextupoles on. Cases 1, 3 and 5: wiggler model D; case 2: wiggler model C. Cases 1,2 and 3: $5^{\text {th }}$ order dynamical map; case 5: linear wiggler map.

With the sextupoles turned on, the dynamic aperture is much reduced, and different wiggler models have only a small effect on the frequency map. The dynamic aperture appears to be limited by a sextupole resonance, at around 10 times the injected horizontal beam size and 6 times the injected vertical beam size. This is likely too small a margin to ensure that injection losses would be negligible, and is likely to be further reduced when other errors are included. 


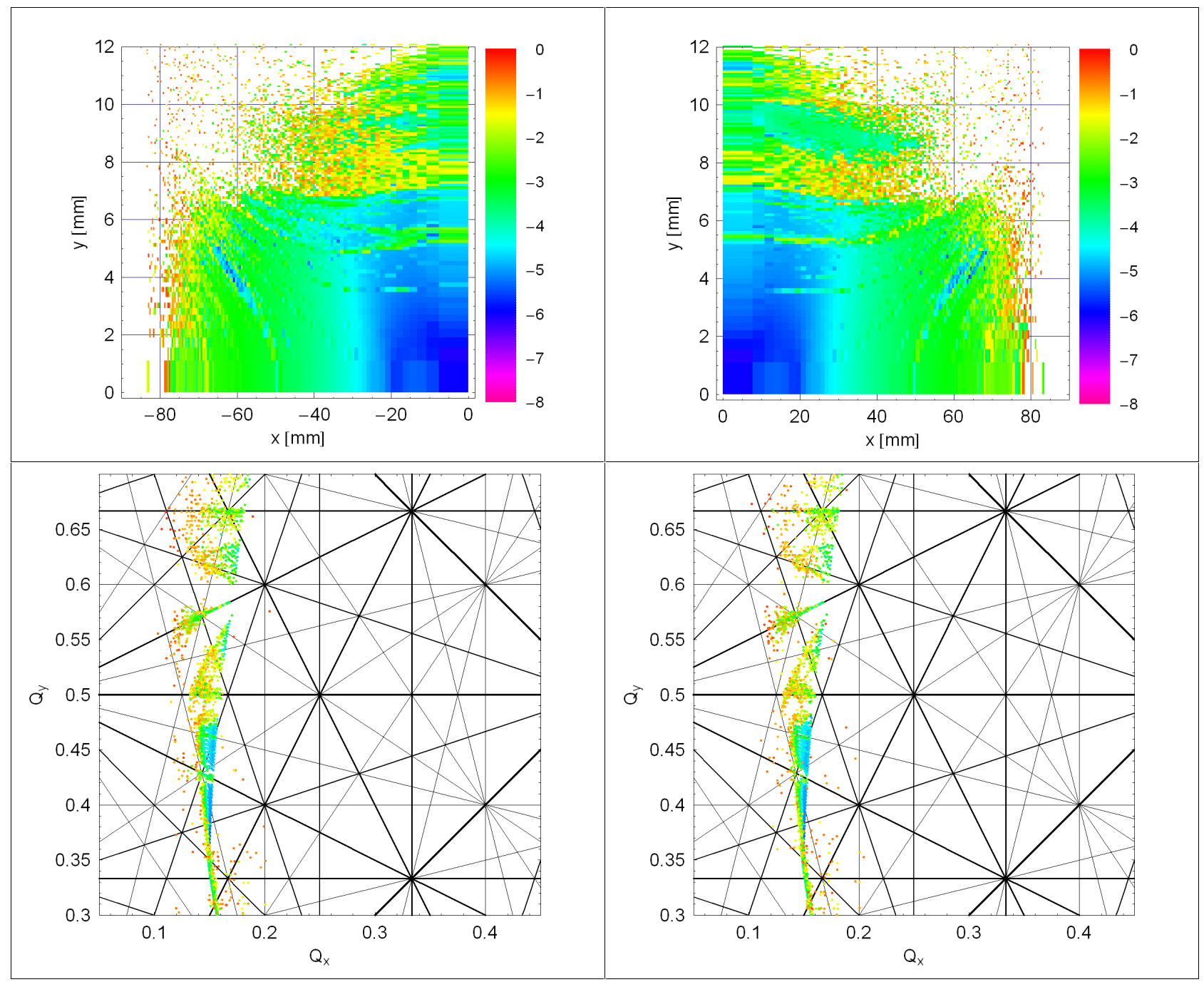

Figure 9: Frequency map plots for Case 1 (wiggler field model D, $5^{\text {th }}$ order dynamical map, sextupoles off) computed with COSY. To be compared with the MARYLIE3.0 computation shown in Figure 10. 


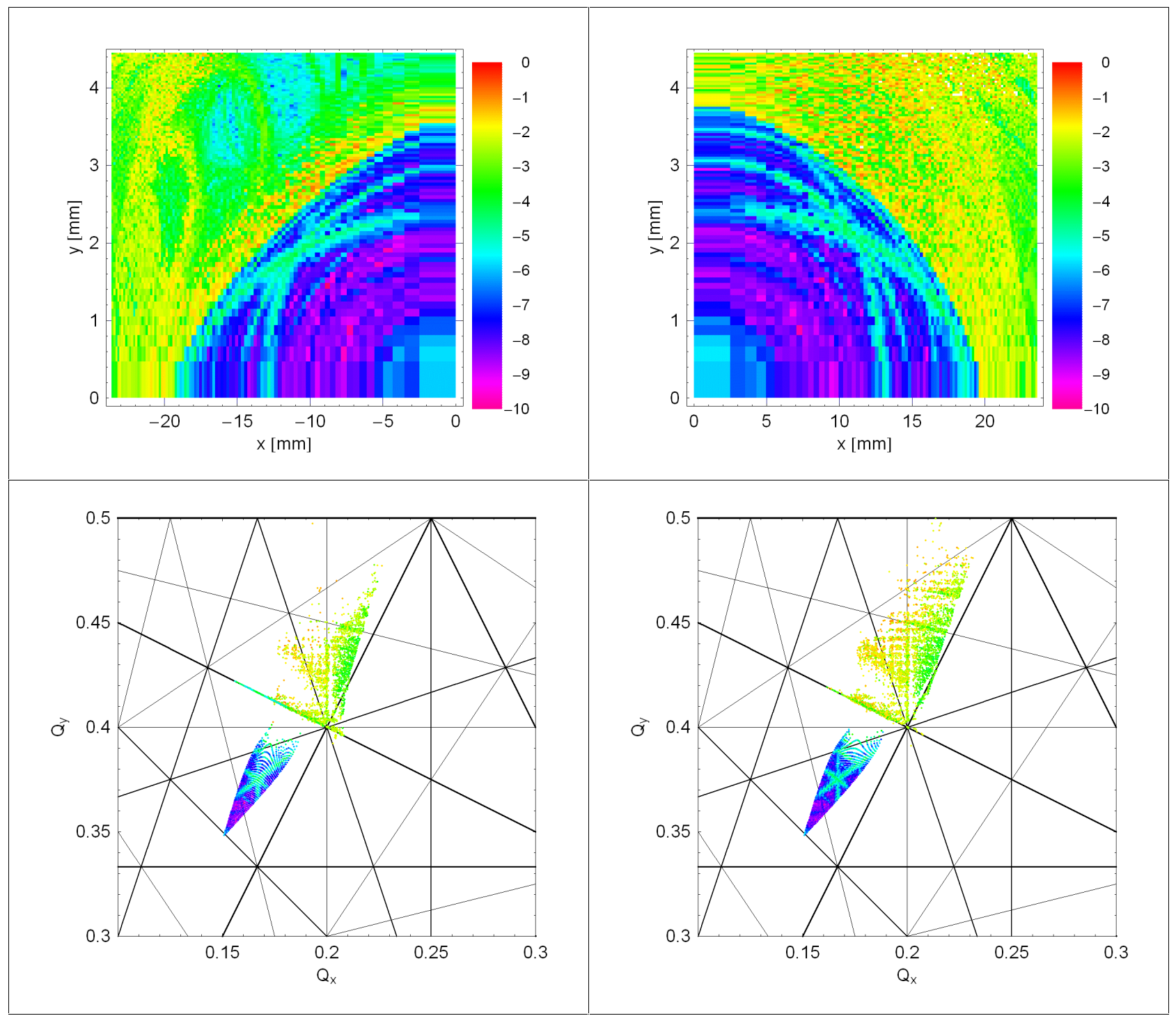

Figure 10: Frequency map plots for Case 1 (wiggler field model D, 3rd order dynamical map, sextupoles off) computed with MARYLIE3.0. To be compared with the COSY computation shown in Figure 9. 


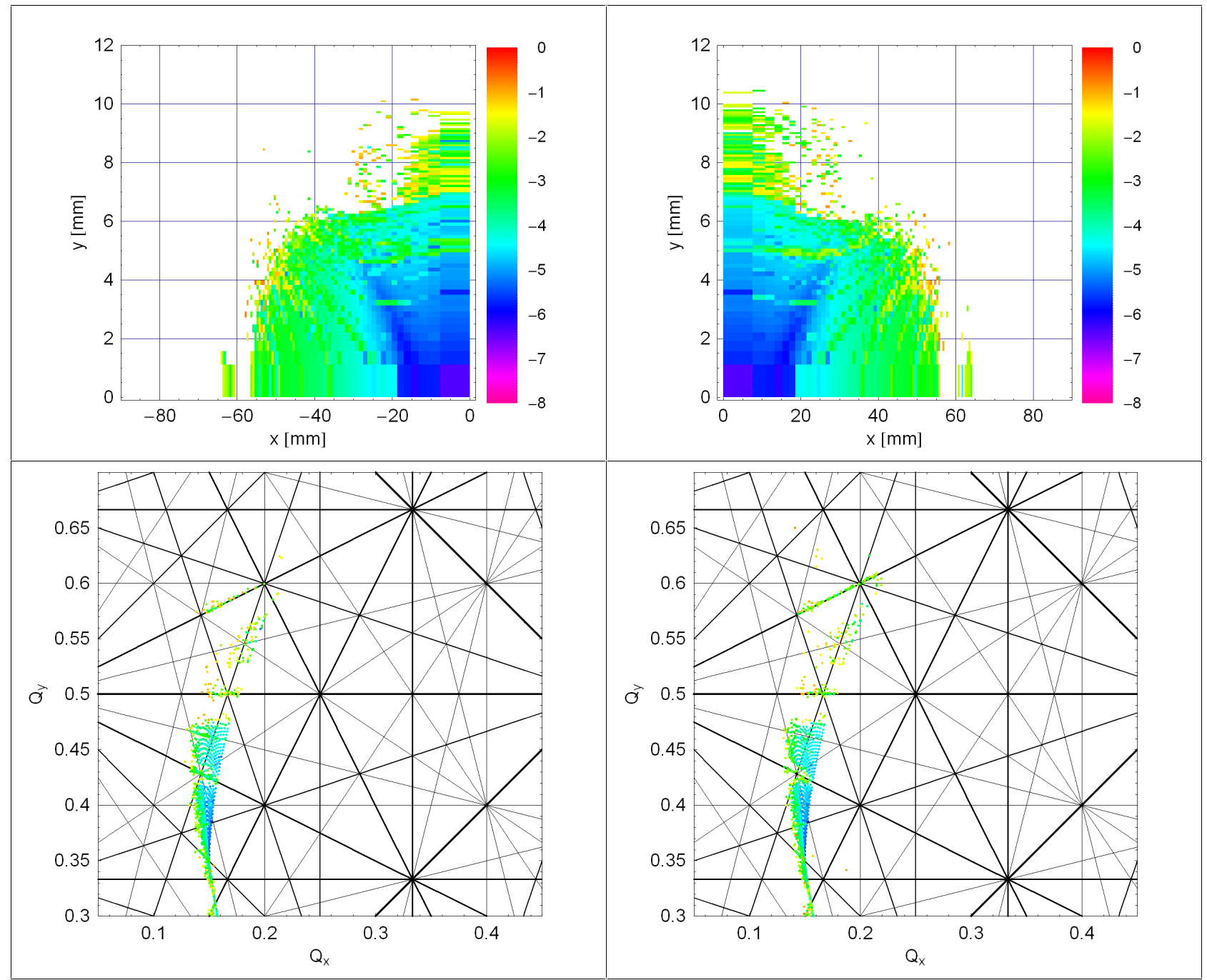

Figure 11: Frequency map plots for Case 2 (wiggler field model $\mathrm{C}$, $5^{\text {th }}$ order dynamical map, sextupoles off) computed with COSY. 


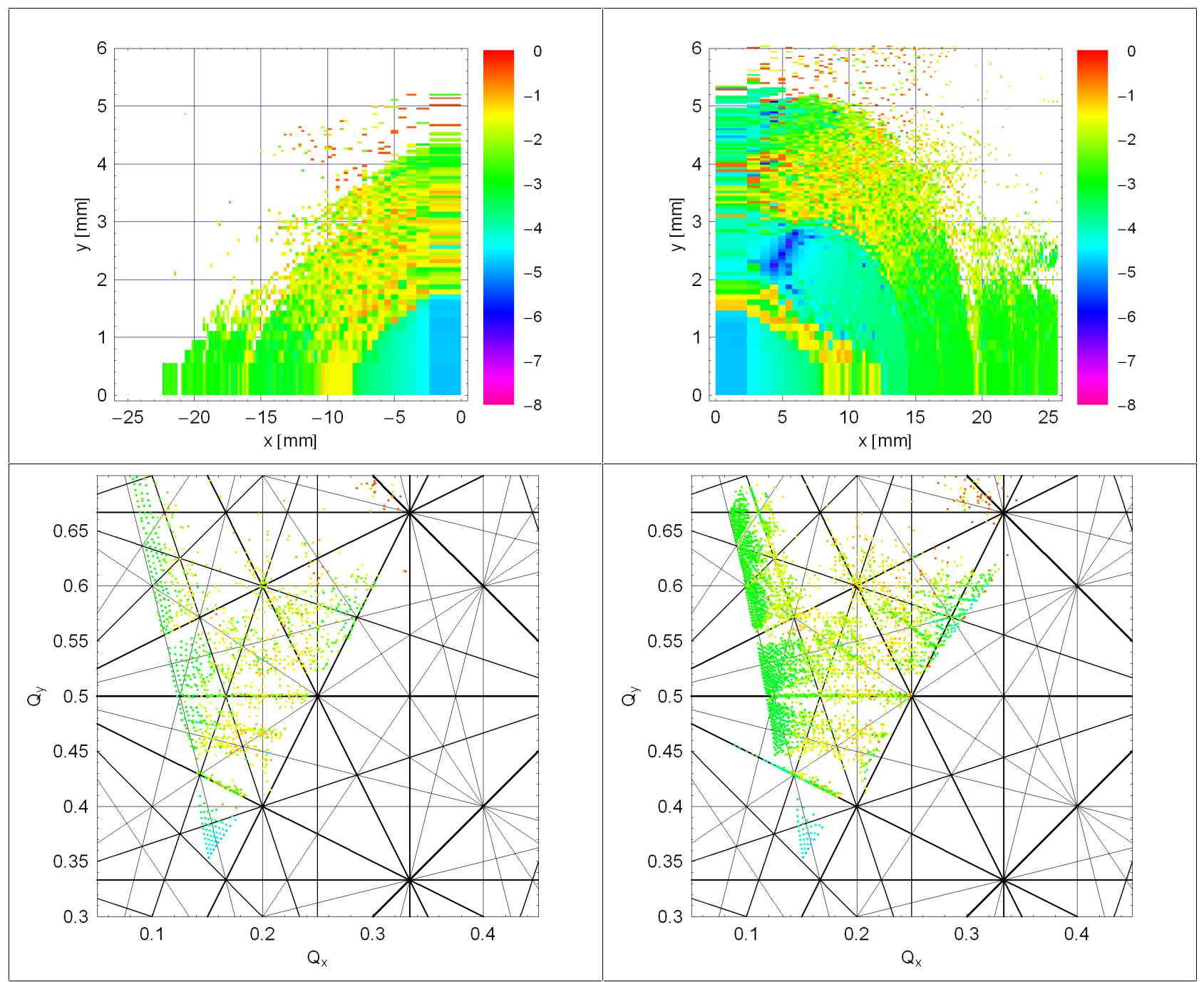

Figure 12: Frequency map plots for Case 3 (wiggler field model D, $5^{\text {th }}$ order dynamical map, sextupoles on) computed with COSY. 


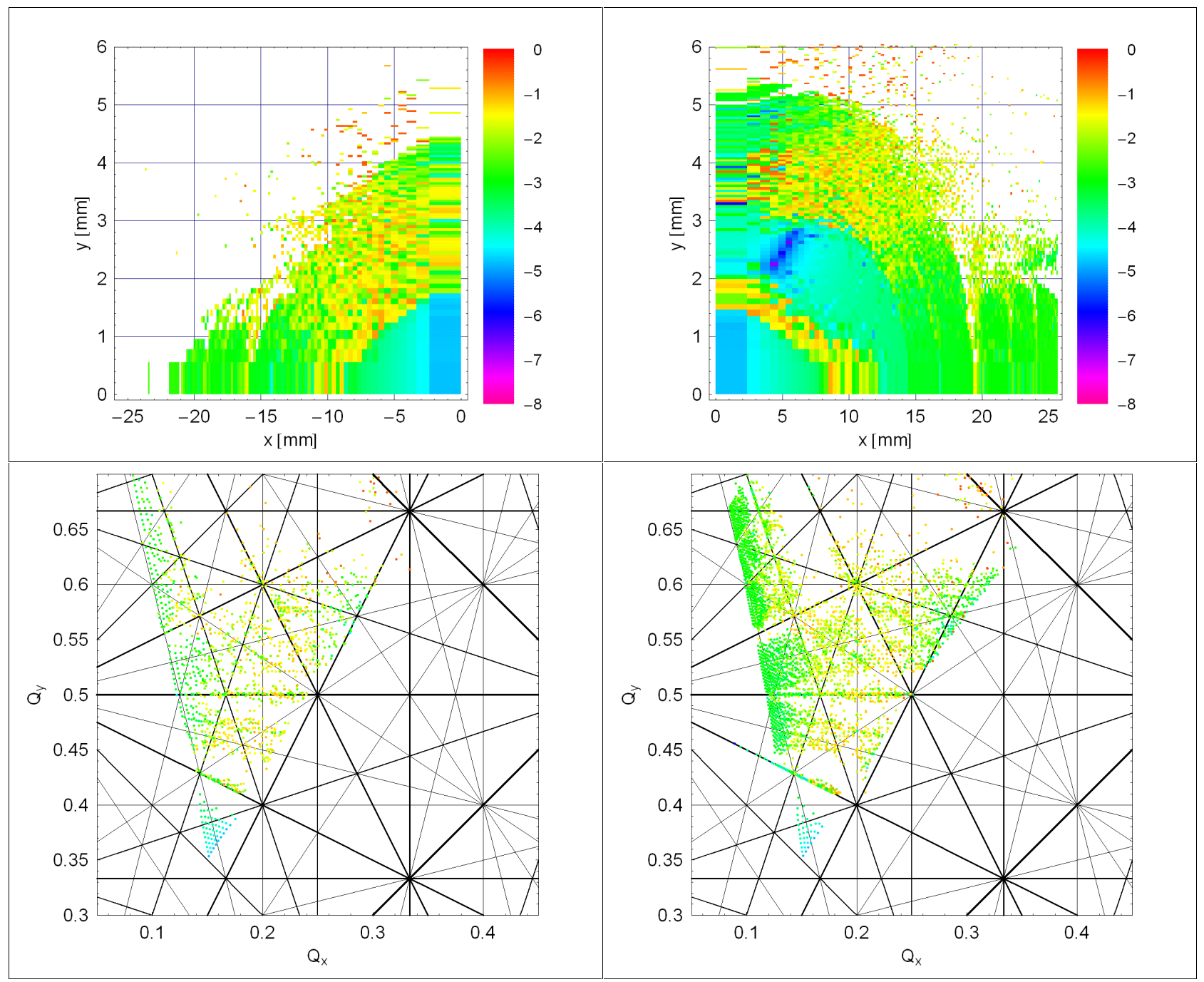

Figure 13: Frequency map plots for Case 4 (wiggler field model D, $3^{\text {rd }}$ order dynamical map, sextupoles on) computed with COSY. To be compared with the MARYLIE3.0 computation shown in Figure 14. 


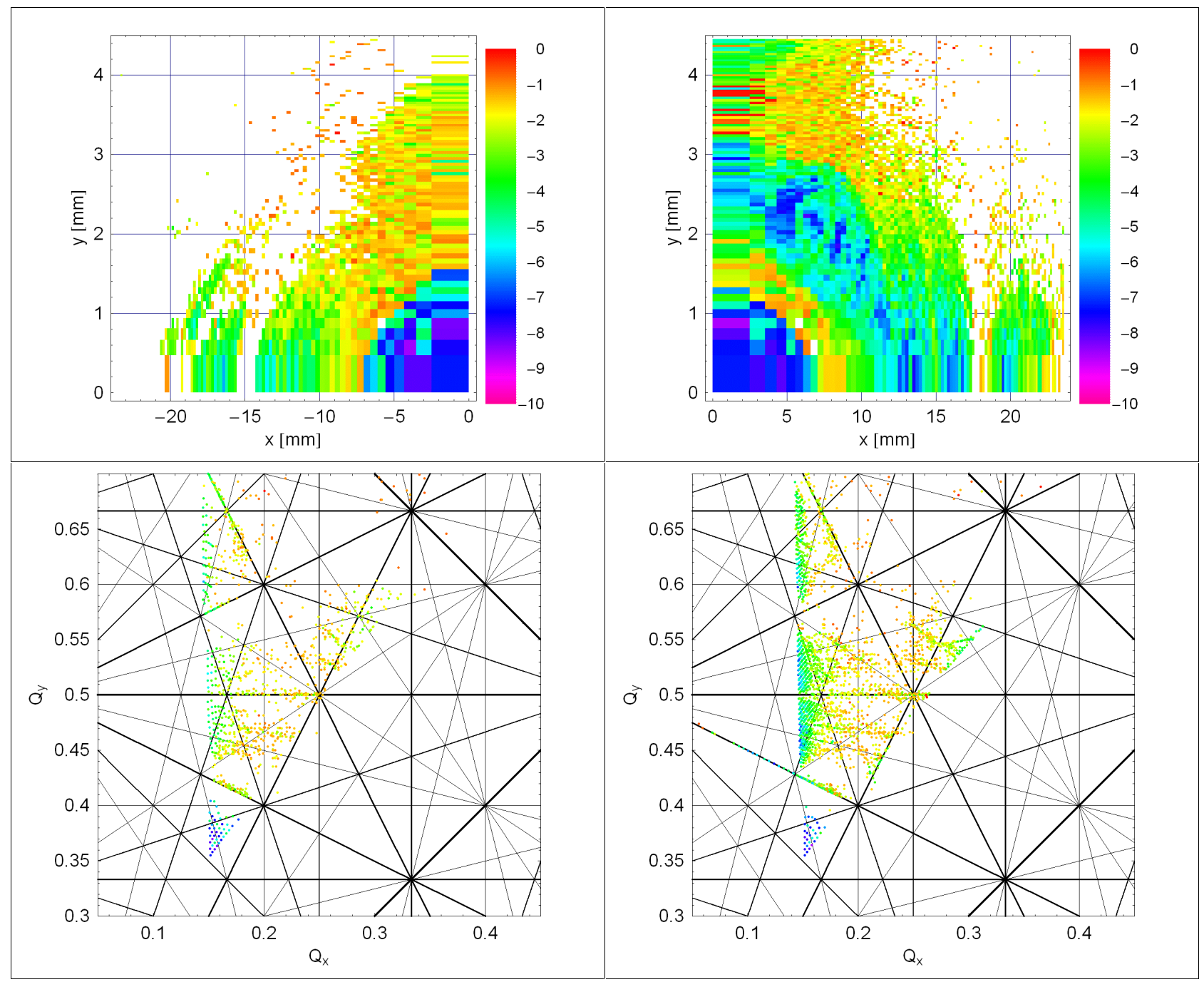

Figure 14: Frequency map plots for Case 4 (wiggler field model D, $3^{\text {rd }}$ order dynamical map, sextupoles on) computed with MARYLIE3.0. To be compared with the COSY computation shown in Figure 13. 


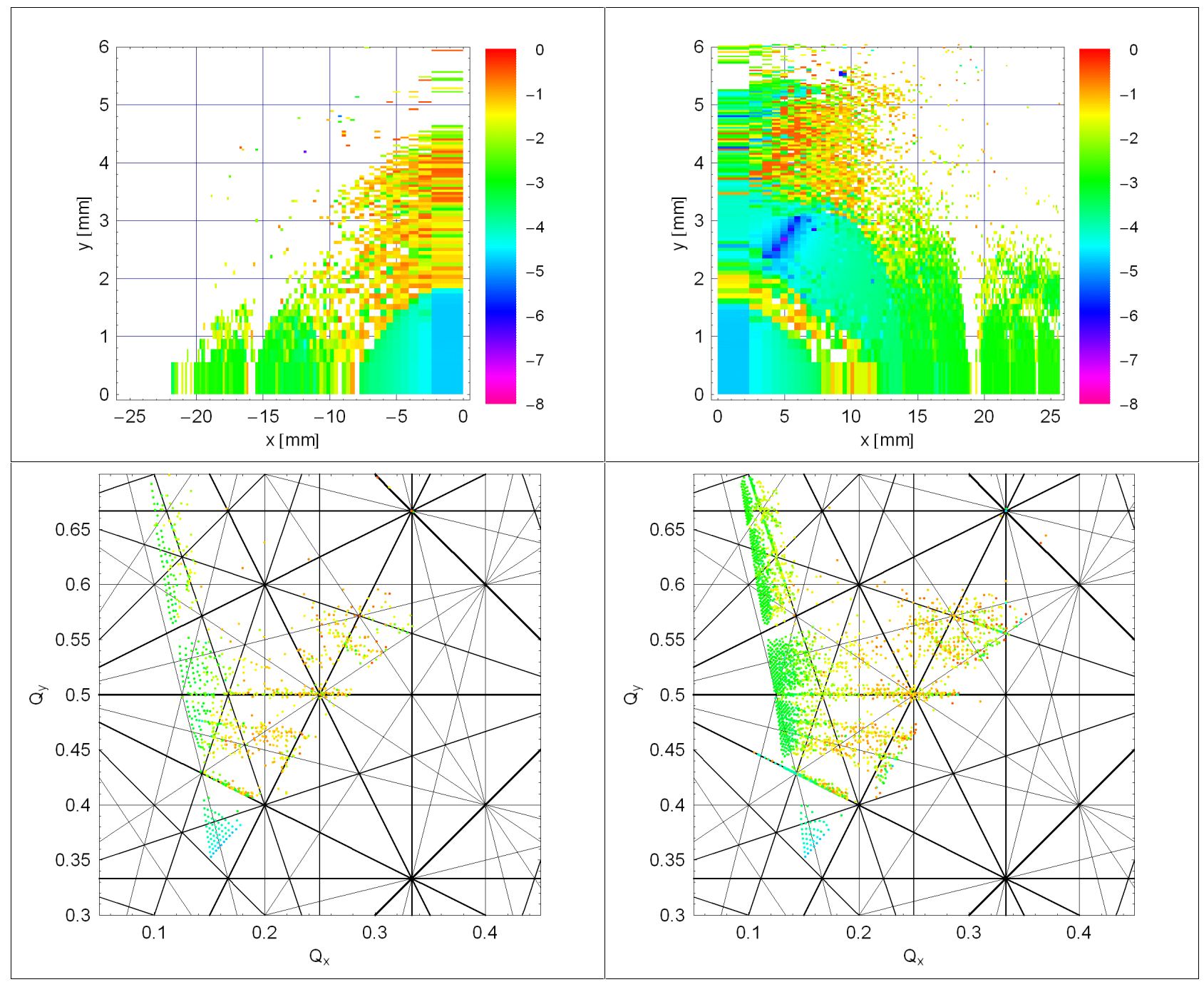

Figure 15: Frequency map plots for Case 5 (wiggler field model D, $1^{\text {st }}$ order dynamical map, sextupoles on) computed with COSY. To be compared with the MARYLIE3.0 computation shown in Figure 16. 


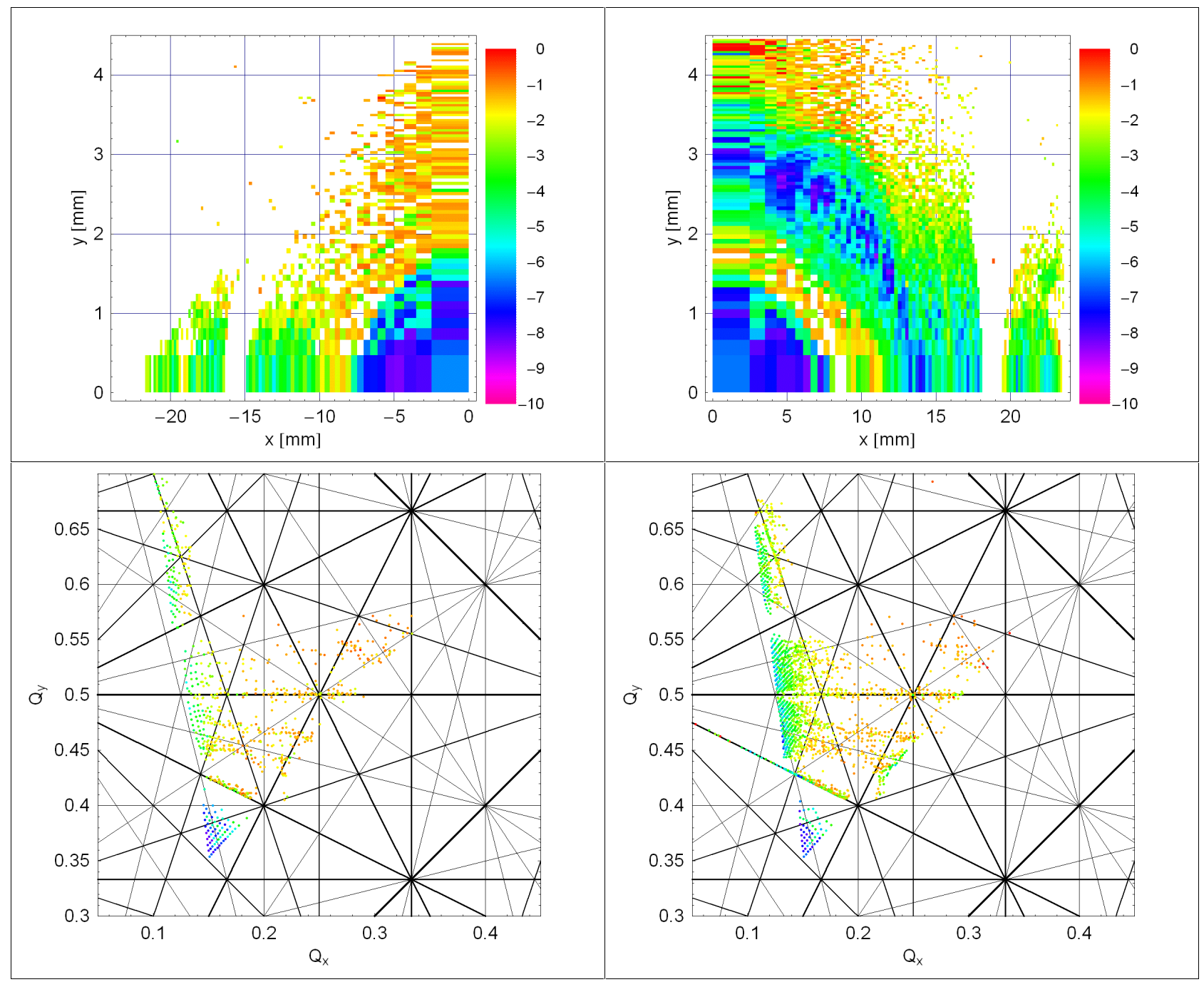

Figure 16: Frequency map plots for Case 5 (wiggler field model D, $1^{\text {st }}$ order dynamical map, sextupoles on) computed with MARYLIE3.0. To be compared with the COSY computation shown in Figure 15. 


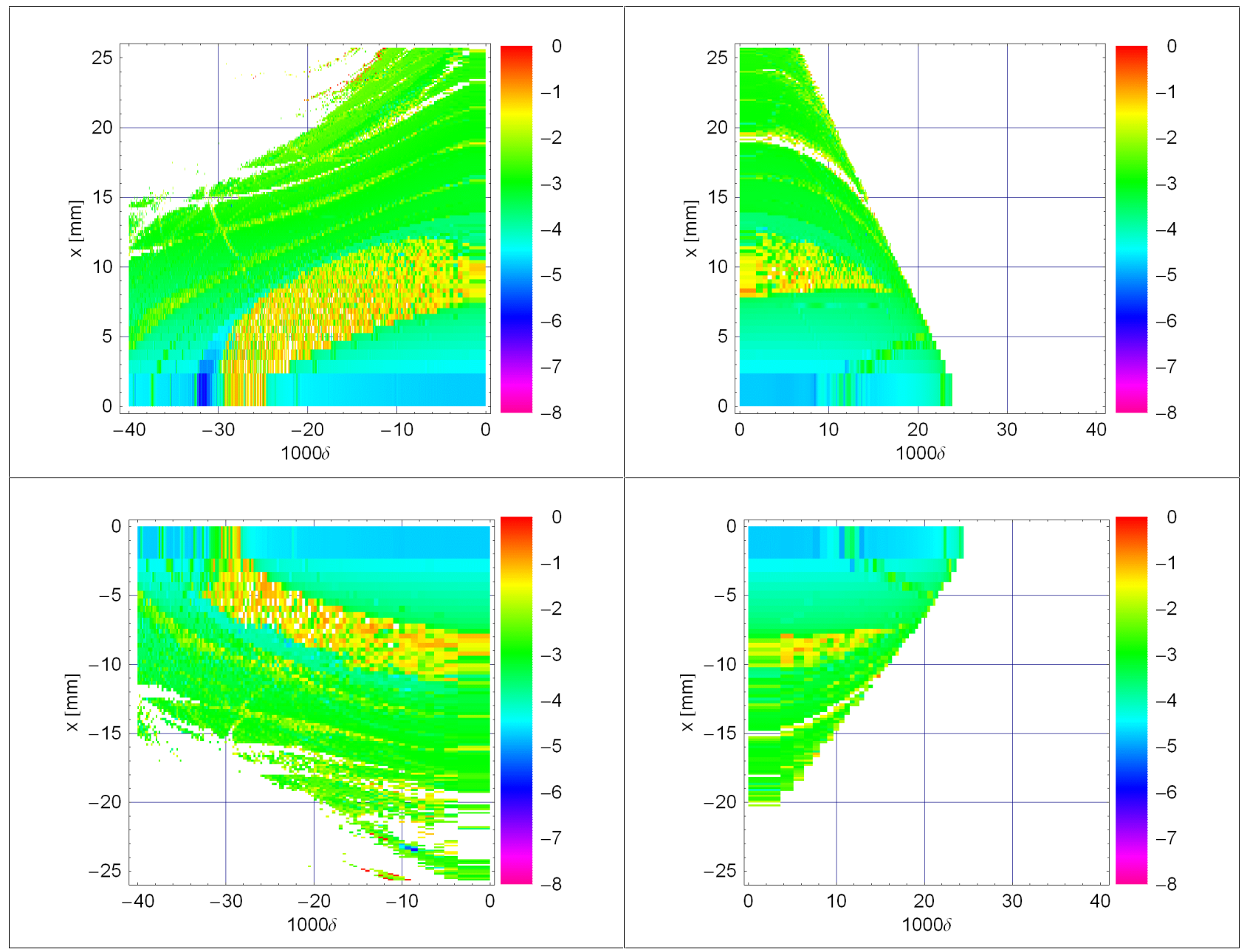

Figure 17: Dynamic aperture plots for Case 6 (wiggler field model D, $5^{\text {th }}$ order dynamical map, sextupoles on, $x$ vs $\delta$ ) computed with COSY. To be compared with the MARYLIE3.0 computation shown in Figure 19. 


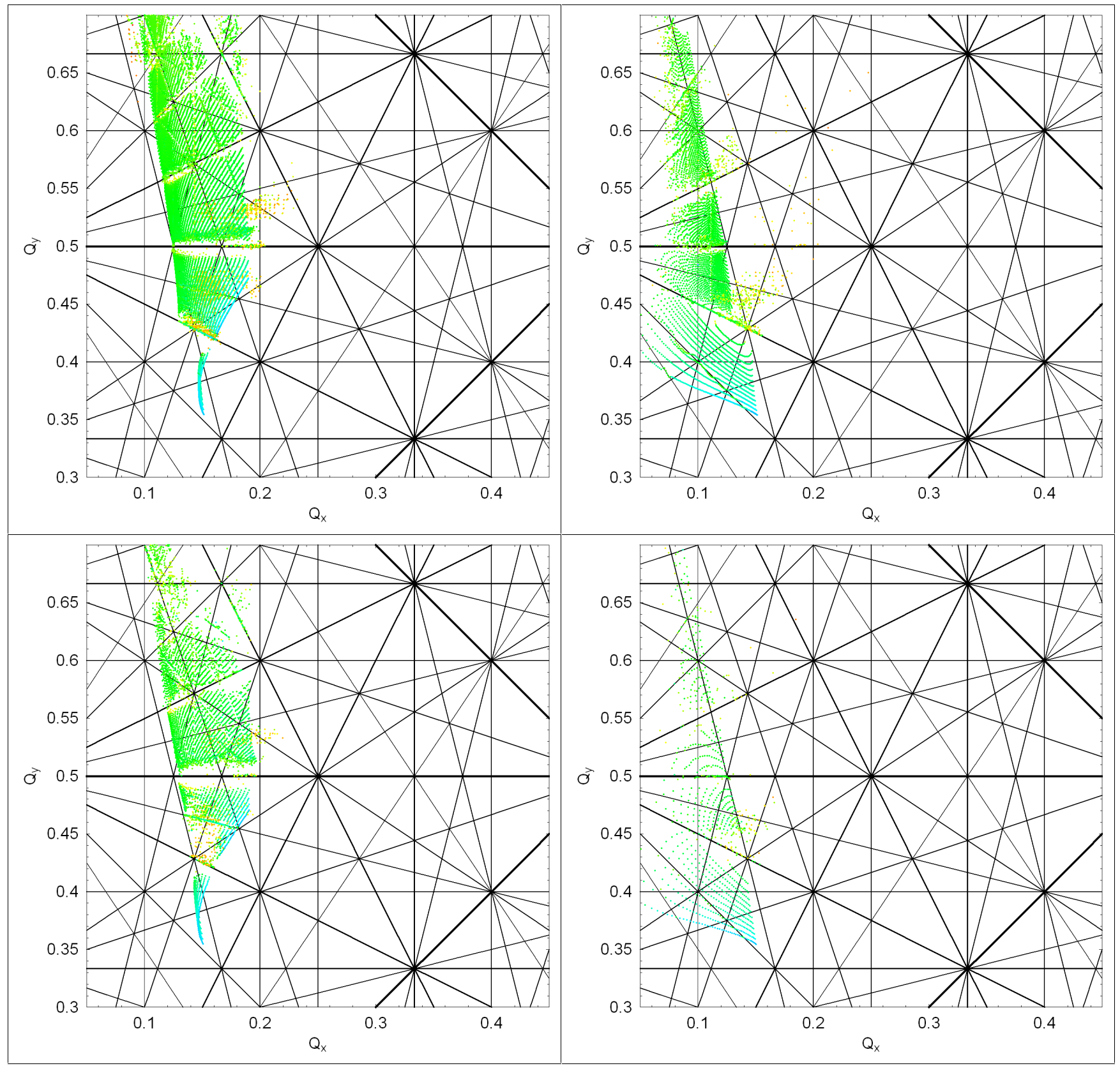

Figure 18: Frequency map plots for Case 6 (wiggler field model D, $5^{\text {th }}$ order dynamical map, sextupoles on, $x$ vs $\delta$ ) computed with COSY. To be compared with the MARYLIE3.0 computation shown in Figure 20. 


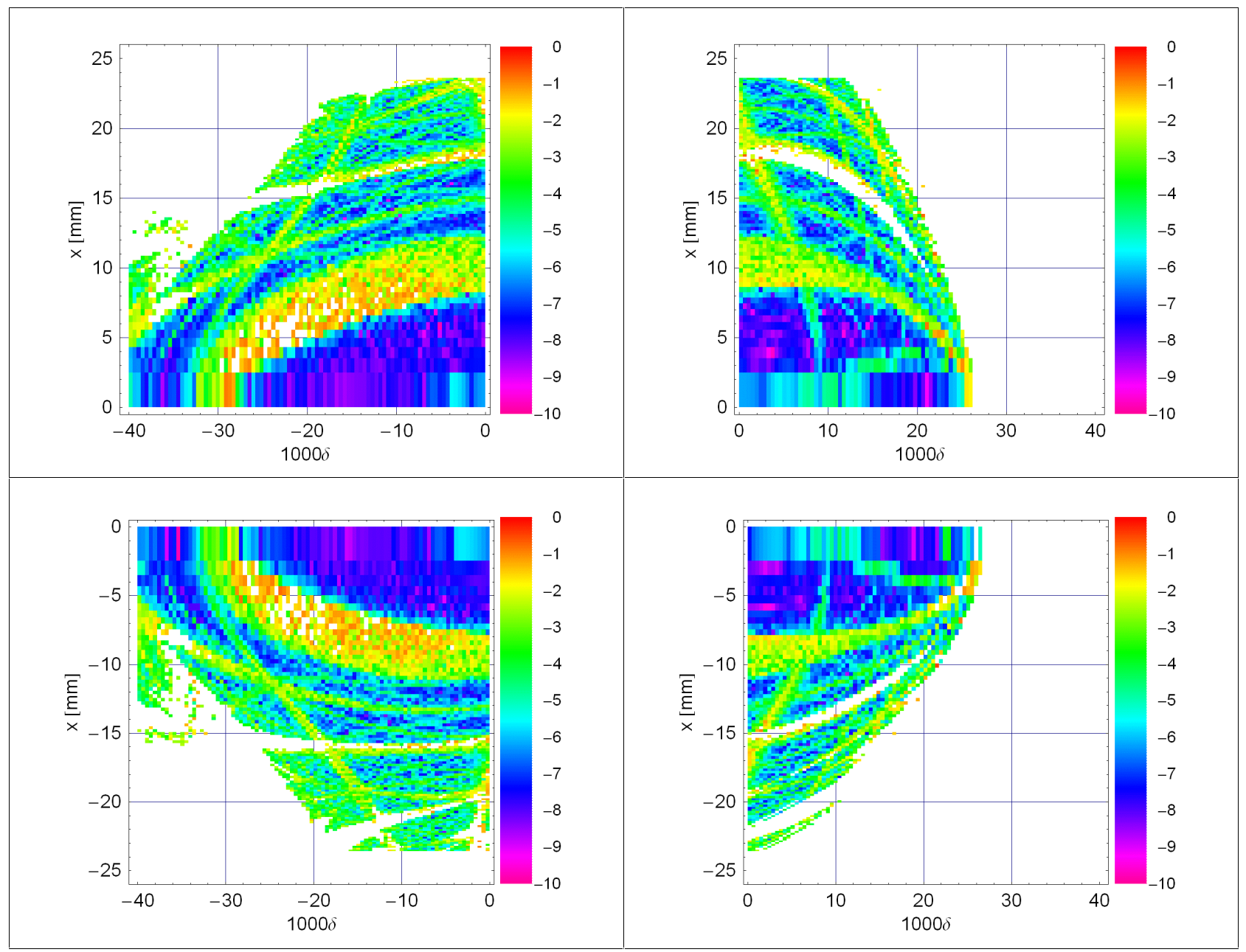

Figure 19: Dynamic aperture plots for Case 6 (wiggler field model D, $3^{\text {rd }}$ order dynamical map, sextupoles on, $x$ vs $\delta$ ) computed with MARYLIE3.0. To be compared with the COSY computation shown in Figure 17. 


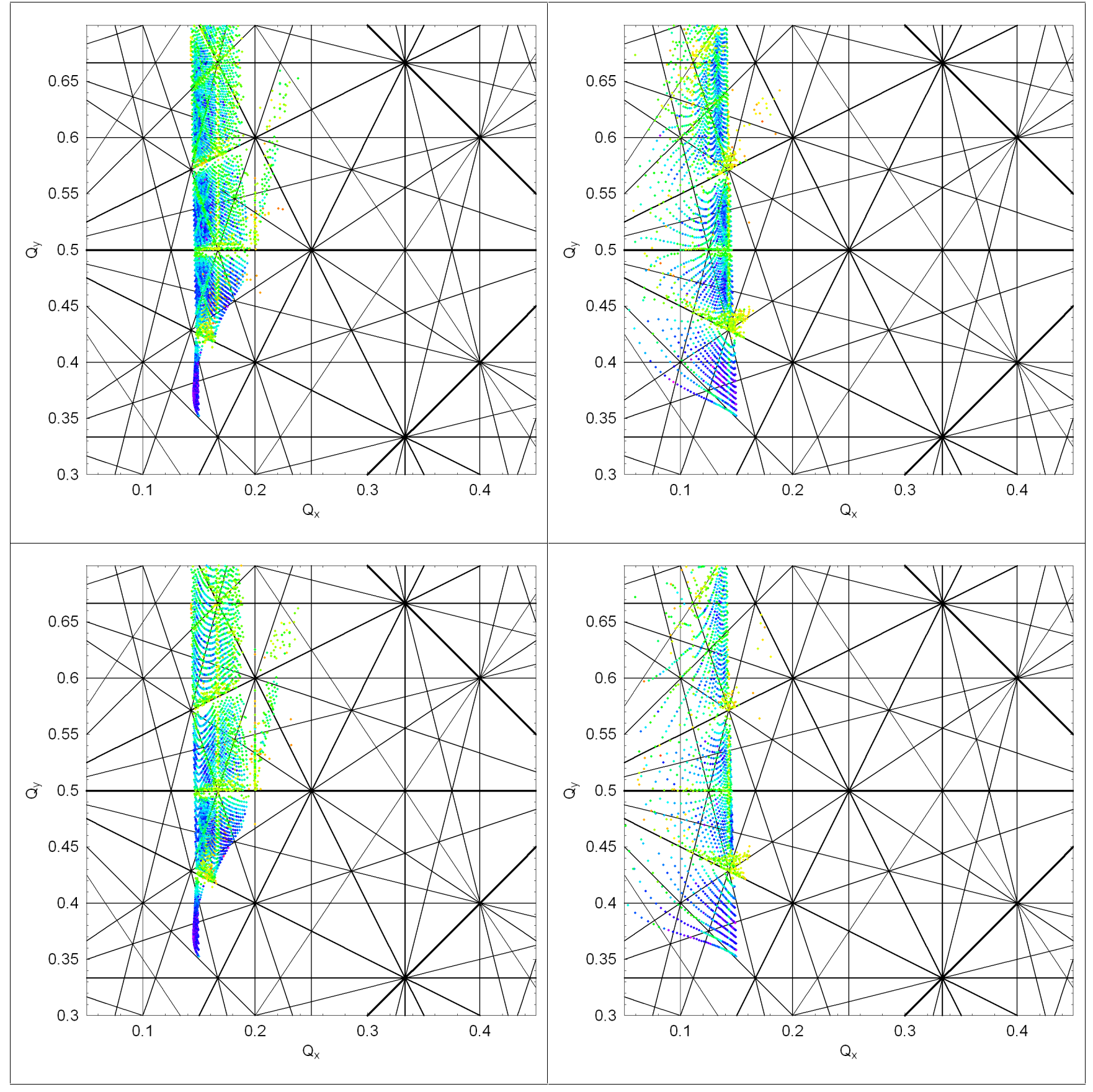

Figure 20: Frequency map plots for Case 6 (wiggler field model D, $3^{\text {rd }}$ order dynamical map, sextupoles on, $x$ vs $\delta$ ) computed with MARYLIE3.0. To be compared with the COSY computation shown in Figure 18.

The dynamic energy acceptance is not symmetric, and appears to range from around $-4 \%$ to around $2.5 \%$. The specified injected full-width energy spread is $1 \%$, so there appears to be some margin here, but again, we can expect some reduction when lattice errors (steering errors, gradient errors and higher-order multipoles in the dipoles, quadrupoles and sextupoles) are included. 
We observe some differences in the frequency maps computed with COSY/MERLIN, compared with the corresponding cases computed with MARYLIE3.0. We see that the MARYLIE3.0 gives somewhat lower diffusion rates than the COSY/MERLIN computation. This is likely to be an effect of the (small) symplectic error in the truncated Taylor map used for tracking in MERLIN. Tracking in MARYLIE3.0 is carried out using a mixed-variable generating function method that ensures symplecticity. We also see some difference in the tune shifts resulting from the wiggler (compare, for example, Figure 13 with Figure 14). This is somewhat surprising, given the good agreement between the maps as shown in Figure 6, and the reasons for the discrepancy are not clear at present. Overall, there is good qualitative agreement between the frequency maps computed with MARYLIE3.0, and those computed with COSY/MERLIN.

\section{Conclusions}

We have collected a set of useful techniques for fitting a magnetic field map, and generating a dynamical map for use in a tracking code. To produce reliable results, it is important to start with good quality field data; even small variations in the field map may produce noticeable differences in the dynamics.

Applied to the wiggler in the NLC Main Damping Rings, it appears that with the present wiggler model, the nonlinear dynamics are dominated by the sextupoles. Although the dynamic aperture is larger (by a factor between 5 and 10) than the injected beam size, and there is some margin in the dynamic energy acceptance, the aperture will be reduced by a variety of errors, including focusing errors, higher-order systematic and random multipole components in the dipoles, quadrupoles and sextupoles, and random field errors in the wiggler. We feel that some improvements to the lattice would be necessary to guarantee injection with negligible beam losses.

Our results have been confirmed by using different codes to generate the dynamical map through the wiggler (MARYLIE3.0 and COSY), and to track through the lattice (MARYLIE3.0 and MERLIN). Although there are some differences between the results from the different codes, there is good overall qualitative agreement. An advantage of the use of the Lie algebra code MARYLIE3.0 for tracking, is that the tracking is symplectic. The Taylor map used in the COSY/MERLIN analysis is not symplectic, and the diffusion rates in tune space are significantly larger.

Frequency Map Analysis gives some indications of the effects limiting the dynamic aperture, and provides some guidance regarding corrective measures. For example, it may be possible to reduce the strength of the sextupole resonance by modifying the chromatic sextupole arrangement, or by using auxiliary sextupoles in the dispersion-free straights. It may also be possible to control the tune shifts with amplitude using auxiliary sextupoles and/or octupoles. These techniques are not always easy to apply, and may even have an adverse effect on the energy acceptance. 


\section{Acknowledgements}

Thanks to J.F. Ostiguy (FNAL) for useful discussions on differential algebra codes and techniques.

\section{References}

[1] M. Woodley and A. Wolski, "The NLC Main Damping Ring Lattice, February 2003", LCC-0113, February 2003.

[2] C. Steier, D. Robin, L. Nadolski, W. Decking, Y. Wu, J. Laskar, "Measuring and Optimizing the Momentum Aperture in a Particle Accelerator", Phys. Rev. E 65, 056506 (2002). See also: D. Robin, C. Steier, J. Laskar, L. Nadolski, "Global Dynamics of the Advanced Light Source Revealed through Experimental Frequency Map Analysis", Phys. Rev. Lett. 85, 558 (2000); C. Steier et al, "Lattice Model Calibration and Frequency Map Measurements at the ALS", Proceedings EPAC 2000.

[3] M. Venturini, "Effect of Wiggler Insertions on the Single-Particle Dynamics of the NLC Main Damping Rings", LBNL-53264, July 2003. 